\title{
Classifying Sensors Depending on their IDs to Reduce Power Consumption in Wireless Sensor Networks
}

\author{
doi:10.3991/ijoe.v6i2.1220 \\ Ayman M. Brisha \\ Beni Suef University, Beni-Suef City, Egypt
}

\begin{abstract}
Wireless sensor networks produce a large amount of data that needs to be processed, delivered, and assessed according to the application objectives. Cluster-based is an effective architecture for data-gathering in wireless sensor networks. Clustering provides an effective way for prolonging the lifetime of a wireless sensor network. Current clustering algorithms usually utilize two techniques, selecting cluster heads with more residual energy and rotating cluster heads periodically, in order to distribute the energy consumption among nodes in each cluster and extend the network lifetime. Clustering sensors are divided into groups, so that sensors will communicate information only to cluster heads and then the cluster heads will communicate the aggregated information to the processing center, and this may save energy. In this paper we show Two Relay Sensor Algorithm (TRSA), which divide wireless Sensor Network (WSN) into unequaled clusters, showing that it can effectively save power for maximizing the life time of the network. Simulation results show that the proposed unequal clustering mechanism (TRSA) balances the energy consumption among all sensor nodes and achieves an obvious improvement on the network lifetime.
\end{abstract}

Index Terms-Wireless Sensor Networks (WSN), Sensor ID, TRSA Algorithm, Hot Spots Problems.

\section{INTRODUCTION}

A wireless sensor network is a collection of sensor nodes interconnected by wireless communication channels. Each sensor node is a small device that can collect data from its surrounding area, carry out simple computations, and communicate with other sensors or with the base station (BS). Such networks have been realized due to recent advances in micro electromechanical (MEMs) systems and are expected to be widely used for more applications such as environment monitoring, home security, and earthquake warning [1]. Despite the infinite scopes of wireless sensor networks, they are limited by the node battery lifetime. Once they are deployed, the network can keep operating while the battery power is adequate. This is critical point to be considered as it is almost impossible to replace the node battery once deployed over an inaccessible area. A Wireless Sensor Network (WSN) is a special type of ad hoc network composed of a large number of nodes equipped with different sensor devices. This network is supported by technological advances in low power wireless communications along with silicon integration of various functionalities such as sensing, communication, and processing. WSNs are emerging as an important computer class based on a new computing platform and networking structure that will enable novel applications that are related to different areas such as environmental monitoring, industrial and manufacturing automation, health-care, and military. Commonly, wireless sensor networks have strong constraints regarding power resources and computational capacity. Topology discovery algorithm for wireless sensor networks finds a set of distinguished nodes to construct the approximate topology of the network. The distinguished nodes reply to the topology discovery probes, thereby minimizing the communication overhead. The algorithm forms a tree of clusters, rooted at the monitoring node, which initiates the topology discovery process. This organization is used for efficient data dissemination and aggregation, duty cycle assignments and network state retrieval. The mechanisms are distributed, use only local information, and are highly scalable. The vision of ubiquitous computing is based on the idea that future computers will merge with their environment until they become completely invisible to the user. Distributed wireless micro sensor networks are an important component of this ubiquitous computing and small dimensions are a design goal for micro sensors. The energy supply for the sensors is a main constraint in the intended miniaturization process. It can be reduced only to a limited degree since energy density of conventional energy sources increases slowly. The abstract sensor is an interval of values that represents the observation provided by a concrete sensor. Finally, the reliable abstract sensor is the interval (or a set of intervals) that always contains the real value of the physical state variable[2].

Wireless distributed micro sensor systems enable fault-tolerant monitoring and control of a variety of applications. Due to the large number of Micro sensor nodes that may be deployed and the long required system lifetimes, replacing the battery is not an option. Sensor systems must utilize the least possible energy while operating over a wide range of scenarios. These include power-aware computation and communication component technology, low-energy signaling and networking, system partitioning considering computation and communication trade-offs, and a power-aware software infrastructure. In this paper we propose and evaluate a (TRSA) algorithm which will wisely organize the network into unequaled clustering depending on their unique ID's, where Clusters Head are selected competition which is unlike (LEACH) as in [3], and with no iteration which differs from (HEED) as in [4]. We put the smaller clusters closed to the Base Station (BS), 
thus they will consume lower energy, and distribute the bigger clusters far from B.S. to save C.H. power consumption. The bigger clusters can use TRSA for routing information between their $\mathrm{CH}$ and $\mathrm{BS}$ via the two relay sensors. The rest of the paper organized as follows: section II covers the cluster techniques in WSN. Section III shows the related work in this area. Section IV introduces the proposed algorithm (TRSA) and inter cluster multi hop routing protocol in detail. Section V analyzes (TRSA) algorithm and shows the simulation results. Finally, section VI concludes this paper with directed for future work.

\section{Clusters IN SENSORS NETWORKS}

Wireless sensor networks pose many challenges, primarily because the sensor nodes are resource constrained. Energy is constrained by the limited battery power in sensor nodes. The form factor is an important node design consideration for easy operability and specified deployment of these nodes, which limit the resources in a node. The protocols and applications designed for sensor networks should be highly efficient and optimize the resources used. Topology discovery algorithm for sensor networks uses data dissemination and aggregation, duty-cycle assignments and network-state retrieval. Network topology as shown in Fig. 1 with white empty node for Normal Sensor (NS), the black field node for Cluster Head, and the big node for the Base Station (BS) which are connected to the internet, provides information about the active nodes, their connectivity, and the reach ability map of the system.

The topology discovery algorithm uses the wireless broadcast medium of communication. The nodes know about the existence of other nodes in their communication range by listening to the communication channel. The algorithm finds a set of distinguished nodes, and by using their neighborhood information constructs the approximate topology of the network. Only distinguished nodes reply to the topology discovery probes, thereby reducing the communication overhead of the process. These distinguished nodes form clusters comprising nodes in their neighborhood. As in Fig. 2 we have nine (9) unequaled clusters, the small size clusters are near to the Base Station (BS), and the big size ones are far from the base station. The Relay Sensors (RS) are in the two sides of the network field. The more suitable place for them is decided depending on equations 1 and 2 . These clusters are arranged in a tree structure, rooted at the monitoring or the initiating node. The tree of clusters represents a logical organization of the nodes and provides a framework for managing sensor networks.

Only local information between adjacent clusters flows from nodes in one cluster to nodes in a cluster at a different level in the tree of clusters. The clustering also provides a mechanism for assigning node duty cycles so that a minimal set of nodes is active in maintaining the network connectivity.

The cluster heads incur only minimal overhead to set up the structure and maintain local information about its neighborhood. Reducing the power consumption of wireless micro sensor networks increases the network lifetime.

\section{RELATED WORK}

Many clustering algorithms have been proposed for wireless sensor networks in recent years. Important and

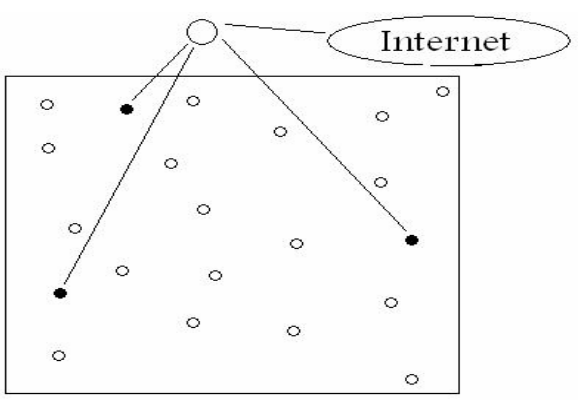

Figure 1. the architecture of WSN in which the sensor nodes are deployed randomly into the interested area (Clusters) depending on their id .

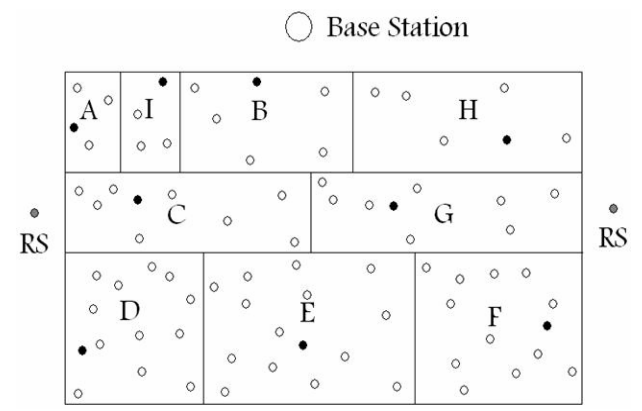

Figure 2. unequaled clusters covered equaled area but the difference is in number of sensors in each cluster.

most relevant papers [3-8] will be reviewed for convenience. In LEACH [3], each node has a certain probability of becoming a cluster head per round, and the task of being a cluster head is rotated between nodes. In the data transmission phase, each cluster head sends an aggregated packet to the base station by single hop. In PEGASIS [6], further improvement on energy-conservation is suggested by connecting the sensors into a chain. To reduce the workload of cluster heads, a two-phase clustering (TPC) scheme for delay-adaptive data gathering is proposed. Each cluster member searches for a neighbor closer than the cluster head within the cluster to set up an energy-saving and delay-adaptive data relay link. HEED [4] extends LEACH by incorporating communication range limits and intra-cluster communication cost information. The initial probability for each node to become a tentative cluster head depends on its residual energy, and final heads are selected according to the cost. In the implementation of HEED [9], multihop routing is used when cluster heads deliver the data to the data sink. The methods mentioned above, require re-clustering after a period of time because of cluster heads' higher workload. In [7], an unequal clustering model is first investigated to balance the energy consumption of cluster heads in multihop wireless sensor networks. The work focuses on a heterogeneous network where cluster heads (super nodes) are deterministically deployed at some pre computed locations, thus it's easy to control the actual sizes of clusters. Through both theoretical and experimental analyses, it is shown that unequal clustering could be beneficial, especially for heavy traffic applications. A similar problem of unbalanced energy consumption among cluster heads also exists in single hop wireless sensor networks. Cluster heads far away from the base station have to transmit packets over longer distances than those of heads closer to the base station. As a result, they will consume more energy. In EECS [8], a distance-based cluster formation method is proposed to produce clusters of un- 
equal size in single hop networks. A weighted function is introduced to let clusters farther away from the base station have smaller sizes, thus some energy could be preserved for long-distance data transmission to the base station. Many energy-aware multihop routing protocols have also been proposed for wireless sensor networks. According to different application requirements, those protocols have different goals and characteristics. In [10], the directed diffusion data dissemination paradigm is proposed. It is based on data-centric routing where the data sink broadcasts the interest. When the sensor has data for the interest, it sends the data along the aggregation tree to the sink. In [11], Gradient-Based Routing (GBR) is proposed as a variant of directed diffusion.

Three different data dissemination techniques (stochastic, energybased, and stream-based schemes) are presented to obtain a uniform distribution of the traffic throughout the whole network. However, these multihop routing protocols may not be applied to applications that require continuous data delivery to the data sink. In [12], the authors investigate an optimization problem of transmission range distribution, i.e., whether nodes can vary their transmission range as a function of their distance to the data sink and optimally distribute their traffic so that network lifetime is maximized. Simulation results show that energy balance can only be achieved at the expense of using the energy resources of some nodes inefficiently. This work reveals the upper bound of the lifetime of a flat sensor network and gives some valuable guidelines for designing multihop routing protocols for wireless sensor networks.

\section{Two Relay SENSOR Algorithm (TRSA)}

Each sensor in the network has a unique identification number ID, two bytes are used for making a unique identifier. The first 10 bits (from 0 to 9) are used for coding(1024) sensors. So we can classify the network to 9 unequal clusters depending on the no. of 1 's in each code as shown in Fig. 3. The remind two bits (all zero and all one) can used as a relay sensors to conduct the information between the far cluster head $(\mathrm{CH})$ and the base station (BS). The six remind bits (from bit no. 10 to bit no.15) could be used to control sensor working and help in power saving to extend the life of the Wireless Sensor Network(WSN) as shown in Fig. 4.

TABLE I.

UNEQUALED CLUSTERS

\begin{tabular}{|c|c|}
\hline Cluster Name & No. of Nodes \\
\hline Cluster A & 10 \\
\hline Cluster B & 45 \\
\hline Cluster C & 120 \\
\hline Cluster D & 210 \\
\hline Cluster E & 253 \\
\hline Cluster F & 210 \\
\hline Cluster G & 119 \\
\hline Cluster H & 45 \\
\hline Cluster I & 10 \\
\hline Total & 1022 \\
\hline
\end{tabular}

We assume that, the sensors are distributed according a homogeneous spatial Poisson process and hence, the number of sensors in a square area of side $2 a$ is a Poisson random variable, $N$ with mean $\lambda A$. We assume that the
Relay Sensors are located at the center of the square area. Thus,

$$
E\left[D_{i} \mid N=n\right]=\int_{A} \sqrt{x_{i}^{2}+y_{i}^{2}}\left(\frac{1}{4 a^{2}}\right) d A=0.765 a
$$

If the total energy spent by the clusterheads to communicate the aggregated information to the processing center is denoted by TE, then

$$
E[T E \mid N=n]=\frac{0.765}{r} n p a
$$

Where "npa" is the total length of the segments from all $\mathrm{CHs}$ to the Relay Sensors. Since data from each sensor has to travel at least one hop, the minimum possible energy consumption in a network with $\mathrm{n}$ sensors is $\mathrm{n}$, assuming each sensor transmits 1 unit of data and the cost of doing so is 1 unit of energy.

In [13] EEUC, the cluster head $(\mathrm{CH})$ chooses a relay node (RN) from its adjacent cluster heads according to the nodes residual energy and its distance to the base station (BS), and the algorithm in [13] is used for selecting the RN.

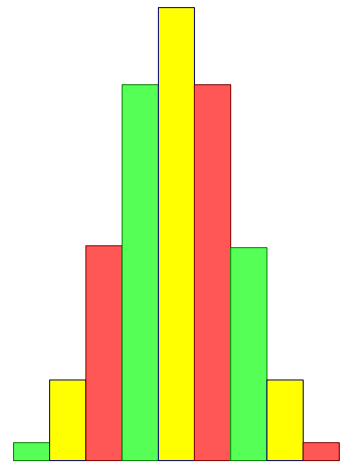

Figure 3. No. of sensor in each Cluster

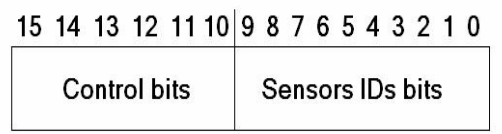

Figure 4. dividing bits for control and addressing sensors

In the proposed algorithm, the two sensors with ID all zero and all 1 are used as relay sensors which proves that two hop for base station is better than one hop. The proposed algorithm is an improved version of that used in [14]. Table 1 shows the number of sensors in each cluster from cluster A to Cluster I, in [14], the authors have assumed that the sensors are equipped with the capability of tuning the power at which they transmit and they communicate with power enough to achieve acceptable signal-to-noise ratio at the receiver. Hereby, it is assumed a network in which the sensors are very simple and all the sensors transmit at a fixed power level; data between two communicating sensors not within each other's radio range is forwarded by other sensors in the network. The authors, in [14], have observed in their simulation experiments that in a network 
with one level of clustering, there is an optimal number of cluster heads that minimizes the energy used in the network. In this paper, results provided in [13] are utilized to obtain the optimal number of cluster heads at each level of clustering analytically.

As in [9], each sensor in the network becomes a cluster head $(\mathrm{CH})$ with probability $p$ and advertises itself as a cluster head to the sensors within its radio range. We call these cluster heads the volunteer cluster heads. This advertisement is forwarded to all the sensors that are no more than $k$ hops away from the cluster head. Any sensor that receives such advertisements and is not itself a cluster head joins the cluster of the closest cluster head. Any sensor that is neither a cluster head nor has joined any cluster itself becomes a cluster head; we call these cluster heads the forced cluster heads. Because we have limited the advertisement forwarding to $\mathrm{k}$ hops, if a sensor does not receive a $\mathrm{CH}$ advertisement within time duration $\mathrm{t}$ (where $\mathrm{t}$ units is the time required for data to reach the cluster head from any sensor $\mathrm{k}$ hops away) it can infer that it is not within $\mathrm{k}$ hops of any volunteer cluster head and hence become a forced cluster head. Moreover, since all the sensors within a cluster are at most $\mathrm{k}$ hops away from the cluster-head, the cluster head can transmit the aggregated information to the processing center after every $t$ units of time. This limit on the number of hops thus allows the cluster-heads to schedule their transmissions. Note that this is a distributed algorithm and does not demand clock synchronization between the sensors. As In [14] an Energy-Efficient Unequal Clustering (EEUC) mechanism for periodical data gathering applications in wireless sensor networks. It wisely organizes the network via unequal clustering and multihop routing. EEUC is a distributed competitive algorithm, where cluster heads are elected by localized competition, which is unlike LEACH [4], and with no iteration, which differs from HEED [4]. The node's competition range decreases as its distance to the base station decreasing. The result is that clusters closer to the base station are expected to have smaller cluster sizes, thus they will consume lower energy during the intra-cluster data processing, and can preserve some more energy for the inter-cluster relay traffic. In the proposed multihop routing protocol for inter-cluster communication, a cluster head chooses a relay node from its adjacent cluster heads according to the node's residual energy and its distance to the base station. Simulation results show that EEUC successfully balances the energy consumption over the network, and achieves a remarkable network lifetime improvement.

\section{OPTIMAL PARAMETERS FOR THE ALGORITHM}

As in $[16,17]$ it is assumed that: a WSN model has the following properties:1) The base station is located far away from the sensors. 2) The nodes have uniform initial energy allocation. 3) The nodes are equipped with power control capabilities 4) Each node senses the environment at a fixed rate and 5) All nodes immobile. 6) Each sensor uses 1 unit of energy to transmit or receive 1 unit of data.7) The communication environment is contention- and error-free; hence, sensors do not have to retransmit any data.8) A distance of $d$ between any sensor and its cluster head is equivalent to $\langle d / r\rceil$ hops.

As in [17] the saving in energy increases as the desity of sensors in the network increases. We use a simplified model shown in [9] for the radio hardware energy dissipation. Both the free space ( $d^{2}$ power loss) and the multi-path fading ( $d^{4}$ power loss) channel models are used in the model, depending on the distance between the transmitter and receiver. The energy spent for transmission of a $l$-bit packet over distance $d$ is:

$$
E_{T x}(l, d)= \begin{cases}l E_{e l e c}+l \varepsilon f_{s} d^{2}, & d \prec d 0 \\ l E_{e l e c}+l \varepsilon_{m p} d^{4}, & d \geq d 0\end{cases}
$$

Where $\mathrm{d}$, is the distance, l-bit packet, $\mathrm{d}^{2}$ for power loss in free space, $\mathrm{d}^{4}$ is power loss in the multi path fading, $\varepsilon_{\mathrm{fs}}$ energy in free space, $\varepsilon_{\mathrm{mp}}$ energy for multi path fading and to receive this message, the radio expends energy is:

$$
E_{R x}(l)=l E_{\text {elec }}
$$

A sensor node also consumes $E_{D A}(\mathrm{~nJ} / \mathrm{bit} / \mathrm{signal})$ amount of energy for data aggregation. It's also assumed that the sensed information is highly correlated, thus the cluster head can always aggregate the data gathered from its members into a single length-fixed packet. As shown in fig. 5 TRSA results is better than LEACH, HEED and EEUC in number of rounds and number of a live sensors.

\section{CONCLUSIONS AND FUTURE WORK}

In this paper, we have assumed that the communication environment is contention and error free. Simulation results show that our unequal clustering mechanism (TRSA) clearly improves the network lifetime over LEACH , HEED and EEUC. Clusters closer to the base station have smaller sizes than those farther away from the base station, thus cluster heads closer to the base station can preserve some energy for the purpose of inter-cluster data forwarding.

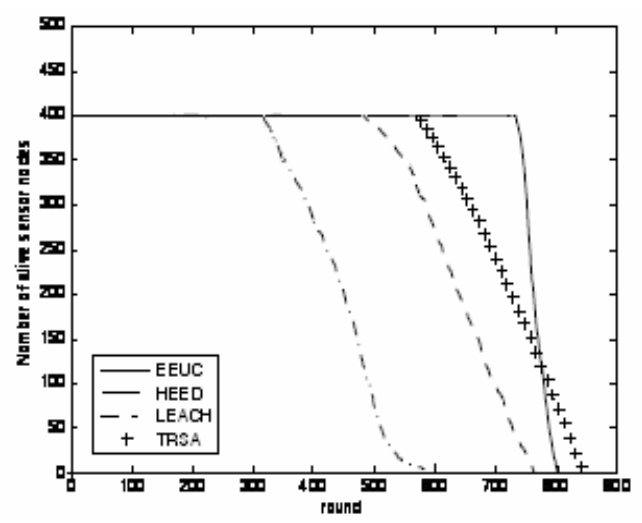

Figure 5. The number of alive sensor nodes over time

\section{REFERENCES}

[1] F. Zhao and L. Guibas, "Wireless Sensor Networks: An Information Processing Approach (Morgan Kaufmann Series in Networking)." San Mateo, CA: Morgan Kaufmann, 2004.

[2] R"OMER, K., BLUM, P., ANDMEIER, L. 2005. Time synchronization and calibration in wireless sensor networks. In Handbook of Sensor Networks: Algorithms and Architectures, I. Stojmenovic, Ed. John Wiley \& Sons, Hoboken, NJ, 199-237. 
[3] W. Heinzelman, A. Chandrakasan, and H. Balakrishnan, "An Ap plication-Specific Protocol Architecture for Wireless Microsensor Networks", IEEE Transactions on Wireless Communications, vol. 1, no. 4, pp. 660-670, 2002. (doi:10.1109/TWC.2002.804190)

[4] O. Younis and S. Fahmy, "HEED: A Hybrid, Energy-Efficient, Distributed Clustering Approach for Ad Hoc Sensor Networks", IEEE Transactions on Mobile Computing, vol. 3, no. 4, pp. 660-669, 2004. (doi:10.1109/TMC.2004.41)

[5] S. Lindsey, C. Raghavendra, K. M. Sivalingam, "Data Gathering Algorithms in Sensor Networks Using Energy Metrics", IEEE Transactions on Parallel and Distributed Systems, vol. 13, no. 9, pp. 924-935, 2002. (doi:10.1109/TPDS.2002.1036066)

[6] W. Choi, P. Shah, and S. K. Das, "A Framework for Energy-Saving Data Gathering Using Two-Phase Clustering in Wireless Sensor Networks", in Proceedings of Int'l Conference on Mobile and Ubiquitous Systems: Networking and Services (MOBIQUITOUS), 2004, pp. 203-212.

[7] S. Soro andW. Heinzelman, "Prolonging the Lifetime of Wireless Sensor Networks via Unequal Clustering", in Proceedings of the 19th IEEE International Parallel and Distributed Processing Symposium (IPDPS), 2005.

[8] M. Ye, C. F. Li, G. H. Chen, and J. Wu, "EECS: An Energy Efficient Clustering Scheme in Wireless Sensor Networks", in Proceedings of IEEE Int'l Performance Computing and Communications Conference (IPCCC), 2005, pp. 535-540.

[9] W. Heinzelman, A. Chandrakasan, and H. Balakrishnan, “An Application-Specific Protocol Architecture for Wireless Microsensor Networks", IEEE Transactions on Wireless communications, vol. 1, no. 4, pp. 660-670, 2002. (doi:10.1109/TWC.2002.804190)

[10] C. Intanagonwiwat, R. Govindan, and D. Estrin, "Directed Diffusion: a Scalable and Robust Communication Paradigm for Sensor Networks", in Proceedings of ACM Mobile Computing and Networking (MOBICOM), 2000, pp. 56-67.
[11] C. Schurgers and M. B. Srivastava, "Energy Efficient Routing in Wireless Sensor Networks", in Proceedings of IEEE Military Communications Conference (MILCOM), 2001, vol. 1, pp. 357-361.

[12] M. Perillo, Z. Cheng, and W. Heinzelman, "On the Problem of Unbalanced Load Distribution in Wireless Sensor Networks", in Proceedings of the IEEE GLOBECOM Workshops on Wireless Ad Hoc and Sensor Networks, 2004, pp. 74-79.

[13] S.G.Foss and S.A. Zuyev, "On a Voronoi Aggregative Process Related to a Bivariate Poisson Process", Advances in Applied Probability, Vol. 28, no. 4, pp. 965-981,1996. (doi:10.2307/1428159)

[14] W. R. Heinzelman, A. Chandrakasan and H. Balakrishnan, "EnergyEfficient Communication Protocol for Wireless Microsensor Networks", in Proceedings of IEEE HICSS, January 2000.

[15] Chengfa Li, Mao Ye, Guihai Chen, Jie Wu " An Energy-Efficient Unequal Clustering Mechanism for Wireless Sensor Networks ", Nanjing University, Florida Atlantic University, 2006.

[16] Jain-Shing Liu, Chun-Hung Richard Lin "Energy-efficiency clustering protocol in wireless sensor networks", Ad Hoc Networks 3 (2005) 371-388. (doi:10.1016/j.adhoc.2003.09.012)

[17] Seema Bandyopadhyay and Edward J. Coyle " An Energy Efficient Hierarchical clustering Algorithm for Wireless Sensor Networks ", School of Electrical and Computer Engineering -Purdue University West Lafayette, IN, USA \{seema, coyle\}@ecn.purdue.edu, 03.

\section{AUTHOR}

Ayman M. Brisha is with the Electronic technology Dep., Faculty of Industrial Education, Beni Suef University, Beni Suef, Egypt, amkhamis69@yahoo.com

Manuscript received February 3, 2010. Published as resubmitted by the author 27 April 2010. 